\title{
Safe delivery service utilization five years preceding the survey in Wayu town, Western Ethiopia
}

\author{
Kababa Temesgen Danusa ${ }^{1,}$, , Gemechu Kejela Jilo ${ }^{2}$ \\ ${ }^{1}$ College of Health Sciences, Department of Midwifery, Arba Minch University, Arba Minch, Ethiopia \\ ${ }^{2}$ College of Health Sciences, Department of Public Health, Arba Minch University, Arba Minch, Ethiopia
}

Email address:

Kabetemesgen@gmail.com (K. Temesgen),gemechukejela86@gmail.com (G. K. Jilo)

\section{To cite this article:}

Kababa Temesgen Danusa, Gemechu Kejela Jilo. Safe Delivery Service Utilization Five Years Preceding the Survey in Wayu Town, Western Ethiopia. Science Journal of Public Health. Vol. 3, No. 1, 2015, pp. 87-92. doi: 10.11648/j.sjph.20150301.25

\begin{abstract}
Background: High maternal mortality rate is one of the major public health concerns in developing countries including Ethiopia. Most of the deaths are caused by factors attributed to pregnancy and childbirth. In Ethiopia only about $10 \%$ of women delivered in health facility with wide regional variation. Objective: The main aim of this study was to assess safe delivery service utilization among mothers who gave birth during five years preceding the survey in Wayu town, of western Ethiopia. Methods and Materials: A cross sectional descriptive study was carried out among women with children less than five years prior to survey conducted from January, 2014 to May, 2014 in Wayu town. Systematic random sampling technique was used to select 371 participants. A pre tested and structured questionnaire was used to collect data. Result: A total of 371 women were included into the study. About $107(28.8 \%)$ of mothers were in the age range of $20-24$ years, $342(92.2 \%)$ were married, $237(63.9 \%)$ were educated and 134(36.1\%) were unable to read and write. The study indicated that $175(47.2 \%)$ of the mothers delivered in health facilities and 196 (52.8\%) mothers gave birth at home. Of mothers who gave birth at home, 52 (14\%) deliveries conducted by Traditional birth attendants and $142(38.3 \%)$ without any assistance of skilled personnel while only two deliveries occurs on the way to health facility. The common reasons for home delivery were sudden onset of labor 125 (63.8\%), presence of TBAs 30 (14.3\%), negative attitude of health workers 2(1\%), and others 41 (20.2\%) which includes; believe in God, fear of high cost services, lack of skilled personnel, poor services, negligence of women. Conclusion and recommendations: Only 47\% institutional delivery service utilization was observed in this study. So, community education about pregnancy, child birth and postpartum and training for all Health Extension Workers are needed. In addition to this, further studies that assess factors associated with institutional delivery both quantitatively and qualitatively are needed.
\end{abstract}

Keywords: Mothers, Save Delivery, Utilization, Wayu Town

\section{Introduction}

Maternal death causes considerable social and personal distress in families, especially because women have the major responsibility in most family matters, including raising children [1].According to estimates of WHO, UNICEF, UNFPA, and World Bank, in 2008, 358,000 maternal deaths occurred worldwide from preventable complications during pregnancy and childbirth. Moreover, 99\% of these deaths occurred in developing countries, and an estimated $87 \%$ occurred in sub-Saharan Africa and South Asia [2]. Similarly, WHO has reported that the proportion of deliveries attended by skilled health providers rose from $58 \%$ in 1990 to $68 \%$ in2008 worldwide, but remained at only about 50\% in Africa[3]. A lifetime risk of maternal death in developing countries is forty times higher than that of the developed countries [4].

Increasing proportion of births delivered in a health facility and under the supervision of health professionals is important to lowering health risks among mothers and children [5].However, in many developing countries the majority of births take place at home. According to analysis of DHS data from 48 developing countries since 2003, in 23 countries more than half of births are reported to take place at home[6]. A study on delivery practices among women in rural India, Punjab, showed that more respondents reported home delivery than institutional delivery [7]. Another study in a semi urban settlement of Zaria Northern Nigeria showed that most women $(70 \%)$ delivered at home and majority of deliveries (78\%) were not supervised by skilled personnel [8].

In Ethiopia, maternal mortality and morbidity levels are 
among the highest in the world. According to 2000 Ethiopia Demographic and Health Survey (EDHS), maternal mortality ratio was 871 maternal deaths per 100,000 live births. The corresponding figure reported in the 2005 EDHS was 673 deaths per 100,000 live births and in 2011 EDHS was 676 per 100,000 live births [6]. Even with this turnaround, the challenges of reducing maternal mortality to the targeted level under MDG are enormous. An explanation for this poor health status among women could be that a considerable proportion of women in the country do not have access to or do not use health services [9].

The proportion of births that occur at home remains high in Ethiopia, and skilled health professionals attend very few births. The proportion of births attended by a skilled health professional and delivered in a health facility has remained around $10 \%$ over the past five years, a far lower level than in other African countries [10].

Strategies have been done in Ethiopia to improve the access to maternal health care since the initiative of safe motherhood program in 1987.This initiative has improved pregnant mothers access to antenatal and slightly delivery care in health facilities. However there is higher difference among the regions despite the higher attendance of antenatal clinics in regions and wore das [6].

Inability to use health facility for giving birth will predispose to maternal mortality and morbidity as a result of many factors. So, proper interventions must be taken to increase delivery in health facilities. If home delivery is not conducted by professionals; it increases the risk of infection, $\mathrm{PPH}$, and transmission of HIV/AIDS to relatives or traditional birth attendants, who conduct deliveries without protective equipment's [3].

Unlike in other parts of Ethiopia, no study has been conducted in wayu town to assess safe delivery service utilization. Therefore, this study was intended to investigate safe delivery service utilization in Wayu town, Oromia regional state of western Ethiopia.The findings of this study help governmental and non-governmental organizations in planning and implementing programs to reduce maternal morbidity and mortality in the study area. It will also help researchers for doing further research.

\section{Methods}

\subsection{Study Design and Set up}

Community based cross sectional study was conducted from January, 2014 -May, 2014in Wayu town which is found in Horro Guduru Wollega zone, Oromia Region. The total population of Wayu town was 19647 (9963 Females and 9684 Males). The Total reproductive age group female was 4322 and the number of mothers who gave birth 5 years before the beginning of January 2014 was 727. All reproductive age group female who were permanent residents of Wayu town was a source population for this study and the study populations were mothers who live in the selected kebeles and gave birth within the last 5 years in Wayu town.
Mothers who gave birth5 years preceding the survey, up to beginning of January, 2014 were included in the study.

\subsection{Sample Size and Sampling Procedures}

Sample size was calculated using single population proportion formula by taking an assumption of proportion of save delivery utilization of $61 \%$ (from study conducted in Holota) [10], 95\% confidence level, 5\% margin of error and an addition of $10 \%$ non-response rate. The final sample size was computed to be 371 mothers.

Wayu town has one kebele and all women who reside in the town and gave birth in the last five years preceding the survey were identified by conducting a pre-survey. Then, households with the study samples were selected by using systematic sampling method (by dividing number of households with eligible women in the town to sample size (by calculating ' $\mathrm{k}$ ' value)) and the first house included in the study was selected by lottery method. Finally, respondents in the selected house hold were interviewed. For households with more than one eligible woman, interview was done by selecting a women using lottery method and in case where there was no eligible woman in the selected house hold, the immediate next house hold was interviewed.

\subsection{Data Collection Tools and Procedures}

Structured interviewer administered questionnaire was adopted from [18] and study conducted on the same topic in different areas and modified based on the study variables and local context. To ensure the quality of data, the questionnaire and consent documents were first developed in English, then translated into local language (Afan Oromo), and finally retranslated into English to check its consistency. Five Health Extension Workers and two BSc nurse supervisors were recruited and trained for three days on ways of data collection. After training, questionnaire was pretested on 5\% of samples who live in kebeles which were not selected for the study before the actual data collection. Supervisors and principal investigator were closely monitored the day-to-day data collection process. Finally data were sorted, checked, entered into the computer and cleaned for analysis.

\subsection{Measurements}

The following operational definitions were used; Safe delivery is giving birth at health facility in a safe and clean way of service. Utilization is the extent to which a given group of people uses particular service in a specific period of time. Skilled attendants are peoples with midwifery skills (midwives, doctors and nurses with additional midwifery education) who have been trained to proficiency in the skills necessary to manage normal deliveries and diagnose, manage or refer obstetric complications.

\section{Data Processing and Analysis}

The data was cleaned, coded, entered, and analyzed using SPSS version 21. Editing of the data occurred after data entry 
by running frequencies and checking for out of range responses. Descriptive statistics was computed to assess safe delivery utilization.

\section{Ethical Consideration}

The study was conducted after getting ethical clearance from Addis Ababa University, College of Health Science, Department of Nursing and Midwifery research committee. Support letter was obtained from Addis Ababa University to Wayu town Health office and from Wayu town health office to the kebele administrative of Wayu town. In addition informed consent was obtained from study participant to confirm willingness for participation after explaining the objective and confidentiality of the study.

\section{Results}

\subsection{Socio-Demographic Characteristics}

A total of 371 mothers who gave birth in the past five years were interviewed making $100 \%$ participation rate. The mean age of the respondents was $28.12 \pm 6.63 \mathrm{SD}$. One hundred seven $(28.8 \%)$ of mothers were in the age range of $20-24$ years. Regarding their marital status, 342 (92.2\%) of respondents were married and only $3(0.8 \%)$ were single. 237 $(63.9 \%)$ of mothers were educated and 134 (36.1\%) were unable to read and write. Out of educated mothers, majority of them, $159(66 \%)$ were attended primary and secondary education. Among respondents, 150 (40.4\%) were housewives, 352 (94.9\%) were Christian and 369 (98.9\%) were Oromo in ethnicity. As to the husband's occupational status, majority, $182(51.1 \%)$ were self-employed. One hundred four, $(28.0 \%)$ of respondents had radio, 27 (7.3\%) had TV and the rest $64.7 \%$ did not possess media of communication. (Table-1).

Table 1. Socio-demographic characteristics of mothers $(N=371)$ in Wayu town, Oromia Region, May, 2014.

\begin{tabular}{ll}
\hline Socio demographic Variables & N (\%) \\
\hline Age of mother & $15(4.0)$ \\
$15-19$ & $107(28.8)$ \\
$20-24$ & $104(28.0)$ \\
$25-29$ & $67(18.1)$ \\
$30-34$ & $42(11.3)$ \\
$35-39$ & $29(7.8)$ \\
$40-44$ & $7(1.9)$ \\
$45-49$ & \\
marital status & $3(0.8)$ \\
Single & $342(92.2)$ \\
Married & $8(2.2)$ \\
Divorce & $4(1.1)$ \\
Widow & $14(3.8)$ \\
Separated & \\
Education status & $134(36.1)$ \\
Unable to read \& write & $39(16.5)$ \\
Non-formal education & $39(16.5)$ \\
Adult education & $58(24.5)$ \\
Primary education & $101(41.5)$ \\
Secondary education and above & \\
Religion & \\
\hline
\end{tabular}

\begin{tabular}{ll}
\hline Socio demographic Variables & N (\%) \\
\hline Muslim & $19(5.1)$ \\
Christian & $352(94.9)$ \\
Ethnicity & $367(98.9)$ \\
Oromo & $4(1.1)$ \\
Amhara & \\
Occupation of respondents & $150(40.4)$ \\
House wife & $34(9.2)$ \\
Peasant & $121(32.6)$ \\
Self employed & $52(14.0)$ \\
Employed by Government & $14(3.8)$ \\
Other & \\
Occupation of Husband (n=356) & $45(12.6)$ \\
Peasant & $1(0.3)$ \\
Pastoralist & $182(51.1)$ \\
Self employed & $125(35.1)$ \\
Employed by Government & $30(0.8)$ \\
Other & \\
Head of the Household & $41(11.1)$ \\
wife & $309(83.3)$ \\
husband & $21(5.7)$ \\
both & $83(22.4)$ \\
Parity & $110(29.6)$ \\
1 & $178(48.0)$ \\
2-3 & $59(15.9)$ \\
4 and above & $177(47.7)$ \\
Family size & $135(36.4)$ \\
less than 3 & \\
7 and above & \\
\hline & \\
\hline
\end{tabular}

\subsection{Utilization of Antenatal Care and Place of Delivery}

Among 371 respondents, $298(80.3 \%)$ had attended antenatal care. Out of 298 women who attended antenatal care, most of them, $244(81.9 \%)$ attended less than four visits and $54(18.1 \%)$ attended more than four visits. Only $73(19.3 \%)$ of respondents never attended antenatal care and the reasons for not attended was given by 58 (79.5\%) mothers as they didn't seen any importance of attending antenatal care.

On one hand, Out of 371 women with children less than 5 years, $175(47.2 \%)$ had skilled worker deliveries in health facilities, $196(52.8 \%)$ deliveries were conducted by unskilled personnel, 52 (14\%) deliveries conducted by Traditional Birth Attendants and 142(38.3\%) occurred at home without any assistance of skilled personnel. And only two deliveries occurred on the way to health facility.

\subsection{Reasons for Unskilled Assistance in Delivery among Women Who Intended to Deliver at Health Facility}

$184(49.6 \%)$ of respondents who had delivered without assistance of skilled attendants were intended to deliver in health facilities and the rest $12(3.2 \%)$ wants to deliver at home

Reasons given by respondents, which hinders them to deliver at health facility, includes; Sudden onset of labor in $118(31.8 \%)$, Lack of transportation to health facility in $12(6.5 \%)$, Bad behavior of health workers in 15(8.2\%), Poor belief to modern medicine in $10(5.4 \%)$ and other responses like culture, religion, fear and lack of knowledge in $29(15.8 \%)$ cases. 


\subsection{Proportion of Women Who Attended Antenatal Clinic and Delivered in Health Facilities}

Out of 298 women attended antenatal care, 155 (88.6\%) delivered in health facilities and 53(27\%) of women who had never attended antenatal care at least once were delivered without assistance of skilled personnel. This indicates that, women who attend antenatal care are more likely to deliver in health facilities compared to those who do not attend.

\subsection{Reasons for Poor Satisfaction with Services at Health Facilities}

Regarding the time taken to reach health facility all respondents spent less than $30 \mathrm{~min}$ to reach the health facility or they reside less than 1 kilometer from health facility. One hundred sixty one, $(92 \%)$ of women were satisfied with the services provided at health facilities while $14(8 \%)$ of them were not satisfied with the services provided at health facilities. Even though most of the respondents were satisfied The main reasons for not satisfied with the services at health facilities in those not satisfied were; short age of drugs and supplies $3(21.4 \%)$, bad behavior of health workers $2(14.3 \%)$, Lack of privacy $2(14.3 \%)$, and Other reasons7(50\%) were as follows; Poor services at health facilities, long waiting time and discomfort.

\subsection{Reasons for not delivering in Health Facilities}

Reasons given by respondents on why they were not delivered in health facilities were, sudden onset of labour in $125(63.8 \%)$, presence of TBAs who are competent and provide friendly services in 30(14.3\%), Negative attitude of health workers to pregnant mothers in $2(1 \%)$, and others like Believe in God, Fear of high cost services, Lack of skilled personnel, Poor services, Negligence of women in 39(19.9\%) cases. (Table 2).

Table 2. Reasons for not delivering in health facility among recent delivered women (n=196) in Wayu town, Oromia Region, May, 2014.

\begin{tabular}{lll}
\hline Reasons for not delivering in health facility & Number & percentage \\
\hline Sudden onset of labour & 125 & 63.8 \\
Negative attitude of health workers & 2 & 1.0 \\
Presence of TBA's & 30 & 15.3 \\
Believing in God & 8 & 4 \\
Fear of high cost services & 5 & 2.5 \\
Lack of skilled personnel & 3 & 1.5 \\
Poor services & 3 & 1.5 \\
Negligence of women & 4 & 2.0 \\
Not safe & 2 & 1.0 \\
Fear of operation & 5 & 2.5 \\
Lack of knowledge & 4 & 2.0 \\
Depend on culture & 3 & 1.5 \\
Don't know & 2 & 1.0 \\
Total & 196 & 100 \\
Reasons for poor satisfaction $(\mathrm{n}=14)$ & & \\
No drugs and supplies & 3 & 21.4 \\
Bad behaviour of health workers & 2 & 14.3 \\
Lack of privacy & 2 & 14.3 \\
Other & 7 & 50.0 \\
Total & 14 & 100.0 \\
\hline
\end{tabular}

\subsection{Socio- Economic Status and Delivery in Health Facility}

Socio economic status was classified according to wealth index using principal component analysis, thereby women were categorized into five quintiles from lowest, second, middle, high and higher which indicates their socio economic status respectively. Forty eight $(50.5 \%)$ of women who can afford the cost of transport when referred to other health facility delivered in health facility compared to114 (41.8\%) who cannot afford.

\subsection{Cultural Factors and Delivery in Health Facility}

Almost all of the respondents $366(24.8 \%)$ reported that there is no any cultural issue concerning delivery or any traditional medicine that must be taken before or after delivery. But, Only $5(1.3 \%)$ of the respondents reported that delivery should be conducted at home and delivery at health facilities are beneficial for those with complications only and traditionally women before delivery apply slight massage by butter on the uterus to enhance the process of delivery and this service was given by traditional birth attendants.

\section{Discussion}

The study results showed that institutional delivery service utilization was $47 \%$. This study finding was higher than the result of 2011 EDHS which is $10 \%$ [12].This might be due to the time gap between the two studies, since 2011 there could be improvement in accessing and utilizing the service. In addition to the above reason, unlike EDHS 2011which include both urban and rural mothers, this study included only Urban mothers in which negative influence of husbands and family members could be lower than Rural Kebeles, and urban mothers might be able to decide on their own health. But, the finding of this study was lower than a study conducted in Holota (61\%) and Asayta Towns (54.4\%) [10, 13]. This difference might be attributable to the economic difference that mothers in this area could have better socio economics and educational status.

In this study, $298(80.3 \%)$ women had attended antenatal care.thisisslightlyhigherthanthatofnationalestimateof $34 \%$ in EDHS -2011 [12] and slightly lower than findings of studies conducted in Holota town $(87.1 \%)$, Jimma town (90\%), Jijiga town $(82 \%)$ and Hadiya zone (86\%) [10, 15-17]. This study also found that, out of 298 women attended antenatal care, most of them $244(81.97 \%)$ attended less than four visits and $54(18.1 \%)$ attended more than four visits.

Home delivery was 196 (53\%) which is not very high compared to study conducted in national EDHS 2011 where $76.4 \%$ of deliveries were assisted by untrained traditional birth attendants or relatives [12]. Out of $196(52.6 \%)$ mothers who delivered at home, $184(49.6 \%)$ were intended to deliver in health facilities and the main reasons for not delivering in health facility were; sudden onset of labour in $12(6.5 \%)$, Lack of transport to health facility in 15(8.2\%), Bad behavior of health workers in $10(5.4 \%)$ and other reasons like culture, religion, fear, lack of knowledge in 29(15.8\%). Similar 
findings had been reported by the study conducted in Gondar, Amhara region where $44.7 \%$ of the respondents reported that labor was short and smooth and the rest $55.3 \%$ of mothers reported preference to give birth in presence of relatives, trust in TBAs, cultural reason and lack of money as reasons for non-use of health facilities [11].

Out of mothers who gave birth at Health facility, 161(92\%) of them were satisfied with the services provided at health facilities while $14(8 \%)$ of them were not satisfied. Even though most of the respondents were satisfied, the main reasons for not satisfied with the services at health facilities by non-satisfied women were; shortage of drugs and supplies $3(21.4 \%)$, bad behavior of health workers $2(14.3 \%)$, lack of privacy $2(14.3 \%)$ and other reasons $7(50 \%)$ were as follows; lack of skilled personnel, poor services at health facilities, long waiting time and discomfort. Similar to this study, a study in Zambia found that $32 \%$ of women said they were not delivered in health facility because of shortage of staff. Even if competent staffs are available, they cannot use their skills without medical supplies and equipment's. The study found that $122(65.2 \%)$ of women were not satisfied with the services at health facilities due to shortage of medical equipment, drugs and supplies [14]

Even though, the classification of wealth index was based on household assets, the finding was Similar to the study done in Sekela Amhara Region. Ninety seven (26.1\%) of the households had monthly income of between $60-408$ ETB and $93(25.1 \%)$ had 694 - 987 ETB monthly income based on quartile classification. One hundred four $(28.0 \%)$ of the respondents had radio and $27(7.3 \%)$ had TV, and the other $64.7 \%$ did not possess media of communication. Due to this majority of respondents haven't get information related to health related information from media and this might affect safe delivery utilization.

Social and cultural factors primarily influence the women's decision making whether to seek care or not rather than affecting women to reach health facility. In this finding, almost all of the respondents, (366) reported that there is no any cultural issue concerning delivery or any traditional medicine that can be taken before or after delivery. But, Only $5(1.3 \%)$ of the respondents reported that delivery should be conducted at home and delivery at health facilities is beneficial for those with complications only.

\section{Strength and Limitations of the Study}

\subsection{Strength}

This study tried to address the prevalence of safe delivery utilization and some of the reasons why most women do not deliver at health facility.

\subsection{Limitations}

Some of the respondents were unable to recall well the various information concerning antenatal care visits, their age and events within the last five years prior to the survey despite the fact that, the most recent births were considered.
Since it is a cross-sectional study, temporal relations could not be assessed. Being descriptive in nature makes this study difficult to test statistical association between outcome and explanatory variables.

\section{Conclusion and Recommendations}

About $47 \%$ institutional delivery service utilization was observed in this study area though $80.3 \%$ of the mothers attended ANC services during their last pregnancy. Out of home deliveries, $52(14 \%)$ deliveries conducted by Traditional birth attendants and 142 (38.3\%) occurred at home without any assistance of skilled personnel while only two deliveries occurred on the way to health facility. The common reasons for home delivery were sudden onset of labor, presence of TBAs, Negative attitude of health workers, Believe in God, Fear of high cost services, Lack of skilled personnel, Poor services and Negligence of women. Based on the results of this study, the following recommendations are made.

Community education about pregnancy, child birth and postpartum, particularly the danger signs of pregnancy, labour and delivery, the actions ensuing complication and the importance of using institutional delivery service at every childbirth should be given to every mother who came to health facility in general and at ANC visits in particular. Training all Health care providers to increase their knowledge, skills and attitudes towards delivery service utilization and to minimize factors associated with low delivery service utilization. Provision of Information, education, communication and empowering mothers is essential, and could help them in decision making regarding their own health and being committed to use the services.

\section{Authors' Contribution}

KT wrote the proposal, participated in data collection, analyzed the data and drafted the paper. GK approved the proposal with great revisions and revised subsequent drafts of the paper. Both authors read and approved the final manuscript.

\section{Acknowledgement}

Authors would like to thanks Addis Ababa University, School of Nursing and Midwifery for their financial support. Deep appreciations have gone to Wayu town administration office and Wayu town health office. Special thanks to all residents of the selected kebele, data collectors, supervisors and all peoples who involved in the study directly or indirectly.

\section{References}

[1] Mekonnen Y, and A. Mekonnen. Utilization of Maternal Health Care Services in Ethiopia. Calverton, MD, USA: ORC Macro. 2002. 
[2] World Health Organization (WHO) U, UNFPA, and the World Bank. Trends in Maternal Mortality: 1990-2008: Estimates Developed by WHO, UNICEF, UNFPA and the World Bank. Geneva, Switzerland: WHO. 2008.

[3] WHO. Maternal mortality in 1995; Estimates developed by WHO/UNICEF, Geneva. 2001.

[4] Hogan MC FK, Naghavi M, Ahn SY, Wang M, Makela SM, et al.. Maternal mortality for 181 countries, 1980-2008: a systematic analysis of progress towards Millennium Development Goal 5. Lancet; 375(May (9726)):1609-23. 2010.

[5] Obaid T, A. Fifteen years after the International Conference on Population and Development: What have we achieved and how do we move forward? . International Journal of Gynecology and Obstetrics. 2009; 106 (2):pp.102-5.

[6] Montagu D, G. Yamey, A. Visconti, A. Harding, and J. Yoong. "Where Do Poor Women in Developing Countries Give Birth? A Multi-Country Analysis of Demographic and Health Survey Data. II PLoS ONE 6(2): 3. 2011.

[7] Kesterton AJ, J. Cleland, A. Sloggett, and C. Ronsmans. Institutional Delivery in Rural India: The Relative Importance of Accessibility and Economic Status. \| BMC Pregnancy \& Child Birth 10(30): 1. 2010.

[8] Idris SH, U.M.D. Gwarzo, and A.U. Shehu. - Determinants of Place of Delivery among Women in a Semi-Urban Settlement in Zaria Northern Nigeria. \| Annuals of African Medicine. 2006;5(2):68-72.

[9] Nations U. Millennium Development Goals Report, United Nations, New York, NY, USA. 2007.
[10] Kidist B YD. Determinants of maternal health care utilization in Holeta town, central Ethiopia. BMC Health Services Research 2013. 2009:13:256.

[11] Mesfin N, H.M. Damen, and M. Getnet. "Assessment of Safe Delivery Service Utilization among Women of Childbearing Age in North Gondar Zone, North West Ethiopia."Ethiop J Health Dev. 2004;18(3):146-52.

[12] International CSAEaI. Ethiopia Demographic and Health Survey 2011. Addis Ababa, Ethiopia and Calverton, Maryland, USA: Central Statistical Agency and ICF International; . 2012.

[13] Melkamu F. Assessment factors of affecting utilization of maternal health care services in Asaita and Dupti Towns, Afar Regional State. 2005.

[14] Shankwaya S. Study to explore barriers to utilization of maternal delivery services in Kazungula district in Zambia. 2009.

[15] Jira C BT. Determinants of Antenatal care utilization in Jimma Town. Ethiopian Journal of Health Sciences. 2005;15(1):4961 .

[16] Zeine A ea. Factors influencing antenatal care service utilization in Hadiya zone. Ethiop J Health Sci 2010 20(2):7582.

[17] Z: Z. Determinants of Antenatal and Delivery care utilization in Town, Somali regional state. JijigaAddis Ababa University; 2009.

[18] Gwamaka S. Utilization and factors affecting delivery in health facility among recent delivered women in nkasi district, Muhimbili University of Health and Allied Sciences,2012 